\title{
Amino Acid Mutations in NS5B Protein among Treatment-naive Genotype 3A Infected Patients
}

\author{
Amina Gul1, ljaz Ali2, Naheed Gul3 and Jawad Ahmed ${ }^{4}$ \\ 1Department of Pathology, Khyber Medical College, Peshawar, Pakistan \\ ${ }^{2}$ Department of Biosciences, COMSATS Institute of Information Technology, Islamabad, Pakistan \\ 3 Department of Medicine, Shifa College of Medicine, Islamabad, Pakistan \\ ${ }^{4}$ Institute of Basic Medical Sciences (IBMS), Khyber Medical University (KMU), Peshawar, Pakistan
}

\begin{abstract}
Objective: To determine the frequency of mutations at specific amino acid positions in full length NS5B gene among chronic HCV genotype 3a infected patients of Peshawar, who had not taken any previous treatment.

Study Design: Cross-sectional descriptive study.

Place and Duration of Study: Institute of Basic Medical Sciences, Khyber Medical University, Peshawar (IBMS, KMU) and Comsats Institute of Information Technology (CIIT), Islamabad from September 2016 to December 2017.

Methodology: HCV genotype determination was carried out among 310 actively infected, treatment-naive patients, using type specific PCR-based genotyping assay. In a total of $162(52 \%)$ HCV genotype 3a isolates, NS5B gene was amplified in $126(78 \%)$ samples using qualitative PCR and sequencing. NS5B gene sequences were analysed for clinically relevant mutations against standard HCV 3 a reference sequence (Isolate NZL1, BAA04609) using MEGA 6 software.

Results: Analysis of HCV NS5B amino acid sequences (aa.1-591), comprising essential motif A-F revealed four novel mutations: A67V, T131I, R374H and M425L in $27(21 \%)$ viral isolates. Mutation D/N244S and D/N310K were found in 14 $(11 \%)$ of the pre-treatment isolates. Mutations at positions 282 and 316 (S282T and C316N/Y) were not identified among studied isolates.

Conclusion: This study reports mutations based on complete NS5B protein of HCV 3a genome that could help predict treatment response among the chronically infected with HCV genotype 3a patients of Peshawar.
\end{abstract}

Key Words: Hepatitis C virus, HCV genotype 3a, NS5B protein.

How to cite this article: Gul A, Ali I, Gul N, Ahmed J. Amino acid mutations in NS5B protein among treatment-naive genotype 3a infected patients. J Coll Physicians Surg Pak 2019; 29(12):1149-1152.

\section{INTRODUCTION}

Hepatitis is a fatal disease of the liver caused by the hepatitis viruses including HCV. People infected with $\mathrm{HCV}$ are prone to develop cirrhosis and hepatocellular carcinoma. ${ }^{1}$ Response rate of antiviral drugs used for the treatment of HCV infection in different geographical regions reflect the diversity of the virus as well as the response of different ethnic groups. Frequency of HCVassociated infection is increasing, and no effective vaccine is so far available. ${ }^{2}$ The aim of antiviral therapy is to accomplish sustained virological response (SVR). ${ }^{3}$ Although treatment with DAAs increases the probability of attaining SVR by $83 \%$ for HCV-3a, various host- and virus-related factors affect therapeutic response. 4

Amino acid mutations in important sub-genomic regions, including the non-structural NS5B protein, have been implicated in predicting response to antiviral therapy. ${ }^{5}$

Correspondence to: Dr. Amina Gul, Department of Pathology,

Khyber Medical College, Peshawar, Pakistan.

E-mail:draminagul@gmail.com

Received: August 09, 2018; Revised: November 29, 2019;

Accepted: November 30, 2019
The HCV NS5B gene, an RNA dependent RNA polymerase, has been labelled as a key target in treatment with interferon (INF) and ribavirin (RBV) as several drugresistance mutations induced by antiviral treatment are found in this region of the genome. 6 Absence of proofreading action of RNA polymerase leads to mutations in the viral genome and poor response to anti-viral treatment. ${ }^{7} \mathrm{HCV}$ NS5B polymerase contains six distinct sequence motifs designated $A-F$, essential for its enzymatic activity making this region an important target for effective antivirals. ${ }^{8}$ Motif A (DTRCFD) and motif C (GDD) are involved in nucleotide binding and catalysis, while motif B (SGVLTTSCDN) harbours an invariant glycine residue, essential for primer positioning. Within motif $D$ (AMTRY), which is involved in NTP binding and catalysis, an arginine residue is present at this locus in all the HCV isolates. ${ }^{8}$ Worldwide mutations induced by INF/RBV combination therapy in HCV NS5B region (D244N, S282T, Q309R, D310N, C316N, A333E and F415Y) have been described.9,10 Mutations in NS5B region have been linked with variable responses to antiviral therapy among HCV-3a as well as other genotype infections. When compared to HCV genotypes, $\mathrm{HCV} 3 \mathrm{a}$, the most abundant type in KP,11 is considered 
responsive to antiviral therapy. However, resistance is emerging even in case of relatively responsive and easyto-treat HCV 3a. ${ }^{12}$ Understanding of underlying molecular mechanisms of viral resistance, such as viral genetic mutations in NS5B region, can help in determining appropriate individualised treatment strategies resulting in better response rates and circumvention of treatment associated side effects of antiviral therapies.

\section{METHODOLOGY}

The study was conducted at Institute of Basic Medical Sciences, Khyber Medical University Peshawar (IBMS, $\mathrm{KMU}$ ) and Comsats Institute of Information Technology (CIIT), Islamabad from September 2016 to December 2017.

Initially, 310 actively infected samples were analysed for identification of HCV genotype using type-specific nested PCR-based genotyping assay. ${ }^{11}$ A total of 162 (52\%) samples identified as HCV genotype 3a were selected for amplification and subsequent sequencing of NS5B gene. HCV NS5B gene was amplified in a qualitative nested $\mathrm{PCR}$ in two overlapping fragments of $0.9 \mathrm{~kb}$ and sequenced in an automated sequencer (ABI Big dye terminator ready reaction $3.1 \mathrm{kit}$ ). Representative sequences were submitted in National Center of Biotechnology and Information (NCBI) Genbank. Translation of NS5B nucleotide sequences into amino acids was performed using MEGA 6 software and subsequently aligned against reference sequence (Accession No BAA04609) using CLUSTAL W programme for mutational analysis. Protein models were generated using molecular graphic system, PyMol. Qualitative variables were expressed as percentages.

\section{RESULTS}

The complete NS5B gene sequence comprised of $1,773 \mathrm{bp}$ (corresponding to nucleotides 7602-9375) coding for 591 amino acids (corresponding residues 2421-3012). To identify amino acid mutations of NS5B gene, HCV NS5B amino acid sequences (aa.1-591), comprising essential motif A-F, were studied in $126(78 \%)$ samples out of a total of $162(52 \%)$ isolates. In total, $36(22 \%)$ samples were excluded from the study as NS5B gene could not be amplified successfully due to low viral load in these patients. Four mutations A67V, T131I, R374H and M425L, were identified among the sequenced viral strains (Table I).

Surface charge analysis of NS5B sequence between $\mathrm{HCV} 3 a$ isolate and reference $3 a$ sequence showed that arginine and methionine, which had mutated to histidine and leucine at position 374 and 425, respectively changed the positively charged surface to partially neutral surface; whereas, the change to valine at position 67 and isoleucine at position 131 had no effect on the surface charge distribution of NS5B protein (Figure 1).
Table I: Frequency of amino acid mutations in HCV NS5B protein (591aa).

\begin{tabular}{l|c|c|c}
\hline $\begin{array}{l}\text { NS5B key } \\
\text { amino acid } \\
\text { position }\end{array}$ & Codon change & $\begin{array}{c}\text { Amino acid } \\
\text { change }\end{array}$ & $\begin{array}{c}\text { Total sequences } \\
\text { analysed } \\
(\mathrm{n}=126)\end{array}$ \\
\hline 67 & GCT-GTA & A-V & 27 \\
131 & ACC-ATT & T-I & 27 \\
244 & GAT-AAT & D-S & 14 \\
310 & GAT-AAA & D-K & 14 \\
374 & CGC-CAC & R-H & 27 \\
425 & ATG-CTT & M-L & 27 \\
282 & ACC-TCC & S-T & - \\
316 & TGT-AAT/TAT & C-N/Y & - \\
\hline
\end{tabular}

A-alanine; $\mathrm{V}$-valine; T-threonine; I- isoleucine; $D$-aspartate; S-serine; $\mathrm{K}$-lysine; $\mathrm{H}$-histidine; $\mathrm{M}$ methionine; L-leucine; C-cysteine; $\mathrm{N}$-asparagine; $Y$-tyrosine; $R$-arginine.

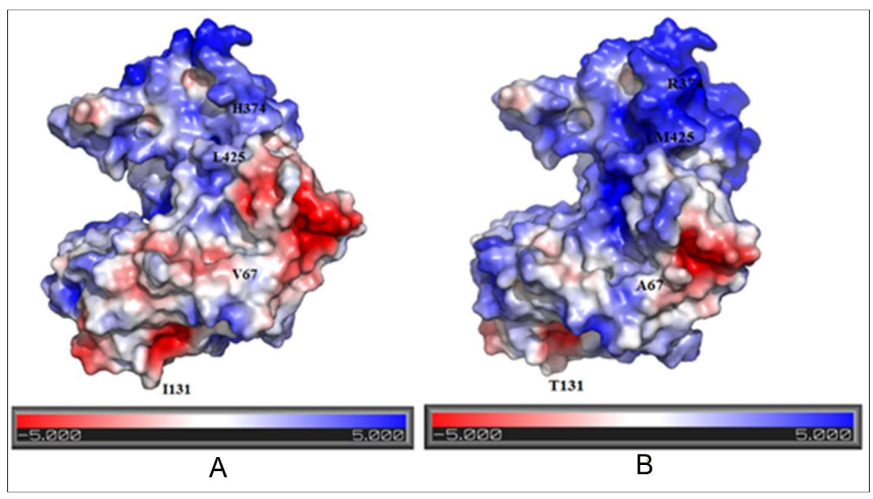

Figure 1: (A) Surface charge distribution of NS5B protein; (B) HCV 3a protein and Ref 3a NZL1; BAA04609. Models were generated using Molecular graphics system pymmmol. Blue and red colour represents positively and negatively charged amino acids, respectively, White colour represents neutral amino acids.

Two mutations D/N244S and D/N310K were found in 14 $(11 \%)$ isolates. Mutation A33R, S282T and C316N/Y were not observed among any of the strains. Mutation Q309R was present in all the isolates. Amino acid residues D220, D225, D318 and D319 comprising the NS5B catalytic pocket (palm domain, motif $A$ and motif $C$ ) were found highly conserved among all the NS5B sequences analysed. Likewise, conserved residues G283, T286, T287 comprising motif B and R345 an essential residue of motif $D$ were shared by all the study patients irrespective of treatment response.

Finally, conserved motif $E$ (residue 361-365) and $F$ (residue 141-160) sequences were also well preserved among all the isolates analysed. A representative number of sequences submitted in NCBI, Genbank can be accessed under the following accession numbers: KU094820, KU094821, KU094822, KU094823, KU094824, KU094825, KU094826, KU094827, KU094828, KU094829, KU094830 and KU094831.

\section{DISCUSSION}

This study reports mutations based on complete NS5B protein of HCV 3 a genome that can predict response to antiviral therapy. Analysis of 591 amino acid positions in NS5B protein of Pakistani HCV isolates was performed and compared to $\mathrm{HCV} 3 \mathrm{a}$ reference isolate (Isolate 
NZL1, BAA04609). So far there is only one report from Pakistan reporting mutations in partial NS5B sequences of HCV 3a patients administered with IFN and RBV combination therapy for six months in which mutations found in our study (A67V, T131I, R374H and M425L) were not found in HCV strains. ${ }^{13}$ One possible reason for this observation could be the partial length of NS5B sequence analysed in their study. However, the same study identified two different mutations D/N244E and D310E linked with non-response to INF/RBV therapy in the studied population. Interestingly we also found a resistant mutation at amino acid residue 244 and 310; but with a different amino acid substitution of serine residue D/N244S and lysine D310K. Worldwide, the mutations induced by INF/RBV therapy in HCV NS5B region (D244N, S282T, Q309R, D310N, C316N, A333E and F415Y) have been reported. However, the results of previously reported studies vary with respect to $\mathrm{HCV}$ genotypes, type of therapy administered, and treatment outcomes. Sugihara et al., observed HCV NS5B amino acid mutations in Japanese type $1 \mathrm{HCV}$ infections but were found insignificant when analysed for their association with treatment response to combination therapy. ${ }^{14}$ Among chronically infected HCV 3a patients from Venezuela and Brazil, two mutations D244N and D310N in NS5B gene were found associated with IFN/RBV therapy, mostly in HCV $3 a$ isolates during the non-treatment observation period.9 Another study by Hamano et al., revealed that amino acid mutations at residues 300-358 of RdRp occurred significantly in those patients reaching an SVR as compared to patients exhibiting non-response.15 The findings of this study were recently investigated in a group of HCV-3a patients from Pakistan. They observed that mutation Q/L309R and $A 333 R$ occurred more frequently in patients reaching SVR. ${ }^{13}$ In contrast, mutation A333R was not found in any of the presently studied isolates analysed; and Q/L309R was present in all isolates, which rules out the possibility of this mutation playing a significant role in predicting treatment response. Presence of mutations among viral strains could be related to the transfer of $\mathrm{HCV}$ types from patients that received treatment. Mutations S282T and C316N/Y have been correlated with resistance to the recently approved DAAs. ${ }^{10}$

Consistent with findings of earlier reports from Pakistan, ${ }^{13}$ the authors could not identify any of the NS5B polymerase resistant mutations among the studied population. Absence of resistant mutations to DAAs might be associated with a higher SVR rates observed in Pakistani $\mathrm{HCV}$ isolates. Moreover, consistent with earlier reports, none of the critical or essential residues were found within NS5B revealed any polymorphisms, indicating the essential role of NS5B polymerase in viral replicative cycle.

\section{CONCLUSION}

Due to essential replicative role of NS5B polymerase in viral lifecycle identification of underlying genetic mutations, it could prove useful in choice of targeted antiviral therapy in case of diverse viral types and subtypes of HCV. In future, whole genome sequencing and comparison of baseline and on therapy viral gene sequences might result in better understanding of antiviral resistance phenomena.

\section{ETHICAL APPROVAL:}

This study was carried out after approval by the Ethics Review Board of Khyber Medical University, Peshawar.

\section{PATIENTS' CONSENT:}

Informed consents were obtained from all the patients to publish their data.

\section{CONFLICT OF INTEREST:}

Authors declared no conflict of interest.

\section{AUTHORS' CONTRIBUTION:}

AG: Study conception and design, manuscript writing, data analysis and interpretation.

IA: Study design, experimentation and data collection. NG: Manuscript writing and data collection.

JA: Critical review.

\section{REFERENCES}

1. Petta S, Craxi A. Current and future HCV therapy: Do we still need other anti-HCV drugs? Liver Int 2015; 35(Suppl 1):4-10.

2. Ansaldi F, Orsi A, Sticchi L, Bruzzone B, Icardi G. Hepatitis C virus in the new era: Perspectives in epidemiology, prevention, diagnostics and predictors of response to therapy. World $J$ Gastroenterol 2014; 20:9633-52.

3. Rizk HH, Hamdy NM, Al-Ansari NL, El-Mesallamy HO. Pretreatment predictors of response to pegIFN-RBV therapy in Egyptian patients with HCV genotype 4. PLoS One 2016; 11: e0153895.

4. Afridi S, Naeem M, Hussain A, Kakar N, Babar ME, Ahmad J. Prevalence of hepatitis $\mathrm{C}$ virus (HCV) genotypes in Balochistan. Mol Biol Rep 2009; 36:1511-4.

5. Marascio N, Torti C, Liberto M, Foca A. Update on different aspects of $\mathrm{HCV}$ variability: Focus on NS5B polymerase. BMC Infect Dis 2014; 14:S1.

6. Li HC, Lo SY. Hepatitis C virus: Virology, diagnosis and treatment. World J Hepatol 2015; 7:1377-89.

7. Blackard JT, Ma G, Limketkai BN, Welge JA, Dryer PD, Martin CM, et al. Variability of the polymerase gene (NS5B) in hepatitis C virus-infected women. J Clin Microbiol 2010; 48:4256-9.

8. Ranjith-Kumar CT, Kao CC. Biochemical activities of the HCV NS5B RNA-dependent RNA polymerase. In: Tan SL, ed. Hepatitis C viruses: Genomes and Molecular Biology. Norfolk (UK) 2006.

9. Jaspe RC, Sulbaran YF, Sulbaran MZ, Loureiro CL, Rangel HR, Pujol FH. Prevalence of amino acid mutations in hepatitis $C$ virus core and NS5B regions among Venezuelan viral isolates and comparison with worldwide isolates. Virol $J$ 2012; 9:214.

10. Ji H, Kozak RA, Biondi MJ, Pilon R, Vallee D, Liang BB, et al. Next generation sequencing of the hepatitis $C$ virus NS5B gene reveals potential novel S282 drug resistance mutations. Virology 2015; 477:1-9. 
11. Gul A, Zahid N, Ahmed J, Zahir F, Khan IA, Ali I. Molecular characterization of hepatitis $C$ virus $3 a$ in Peshawar. BMC Infect Dis 2016; 16:163.

12. Muir AJ. The rapid evolution of treatment strategies for hepatitis C. Am J Gastroenterol 2014; 109:628-35.

13. Ali M, Rehman IU, Idrees M. Emergence of genetically variant Hepatitis $C$ virus population in response to increased antiviral drug pressure, Pakistan. Virus Genes 2014; 48:543-9.

14. Sugihara K, Orito E, Tanaka Y, Kato T, Lau JY, Ohno T, et al.
Variations in the viral NS5B region in Japanese patients with chronic hepatitis $C$ virus genotype $1 \mathrm{~b}$ infection. No specific amino acid substitution was identified as determinants of treatment response to interferon/ribavirin combination therapy. Intervirology 2006; 49:319-26.

15. Hamano K, Sakamoto N, Enomoto N, Izumi N, Asahina $\mathrm{Y}$, Kurosaki M, et al. Mutations in the NS5B region of the hepatitis C virus genome correlate with clinical outcomes of interferonalpha plus ribavirin combination therapy. J Gastroenterol Hepatol 2005; 20:1401-9.

$\cdots \cdot \hat{n} \cdot \ldots$ 